\title{
Longitudinal Light Clock and Zeno's Paradox
}

\author{
Joshua Fixelle and K. Austin Johnson \\ Department of Physics \\ The Pennsylvania State University, Abington \\ 1600 Woodland Road \\ Abington, Pennsylvania 19001, USA
}

Received: July 18, $2010 \quad$ Accepted: November 24, 2010

\begin{abstract}
Introductory college text books consider time dilation through the derivation of the transverse light clock. We consider the case of the longitudinal light clock and derive the time dilation formula of special relativity. Our methods yield the same result as derived by the transverse light clock, and help explain the concept of failure of simultaneity.
\end{abstract}

\section{INTRODUCTION}

The usual treatment for the relativistic light clock is that of the transverse light clock, which is present in most college physics textbooks-such as [1-3]. Two inertial frames of reference are defined: an observer (frame 1) who sees a space ship move by with speed $v$, and a frame attached to the ship (frame 2). A transverse light clock travels on board the ship in the standard presentation. This operates by a beam or burst of light reflected between two aligned mirrors which are positioned perpendicular to the direction of motion. For the observer at rest with respect to the light clock, the period observed is $T_{0}=2 \mathrm{~L} / \mathrm{c}$, where $L$ is the distance of separation between the two mirrors. As derived in [1], the period observed in frame 1 would be the perpendicular velocity component of the light beam, obtained via the Pythagorean theorem. The period would then simplify to the equation $T^{\prime}=\gamma^{2} \mathrm{~L} / \mathrm{c}$. When $\mathrm{T}_{0}$ is substituted, the equation for relativistic time dilation in special relativity is obtained: $T^{\prime}=$ $T_{0} \cdot \gamma$.

Would the results be any different for the longitudinal light clock? This is the question that we address in this paper. Intuitively we know that the derivation will not be as straight forward as that of the transverse light clock-which likely is why textbooks consider the transverse over the longitudinal.

\section{LONGITUDINAL LIGHT CLOCK DERIVATION}

The transverse light clock is usually derived by accounting for half of the period and multiplying the result by two due to symmetry. In the longitudinal light clock, this cannot be done. Imagine two mirrors (mirror $A$ and mirror $B$, with $B$ a distance $L$ to the right of $A$ ), both mirrors moving at constant velocity $v$ to the right (as measured in frame 1). Mirror A has a way to produce light and receive it-the time from production to reception is one period of the clock. Now thinking about this from the perspective of an observer in frame 1, when the light beam is produced it will begin to head towards mirror B; however mirror B will move away from the light beam with speed $v$, thus requiring the light beam to travel a distance greater than $L$. Once the light beam hits mirror $B$ and reverses direction, mirror $A$ will be heading towards the light beam at speed $v$, thus requiring the light beam to travel a shorter distance than $L$. Thus, intuitively, the time for the light to travel in each direction will not be equal to each other in frame 1 , and will not be equal to half of the transverse light clock period. 


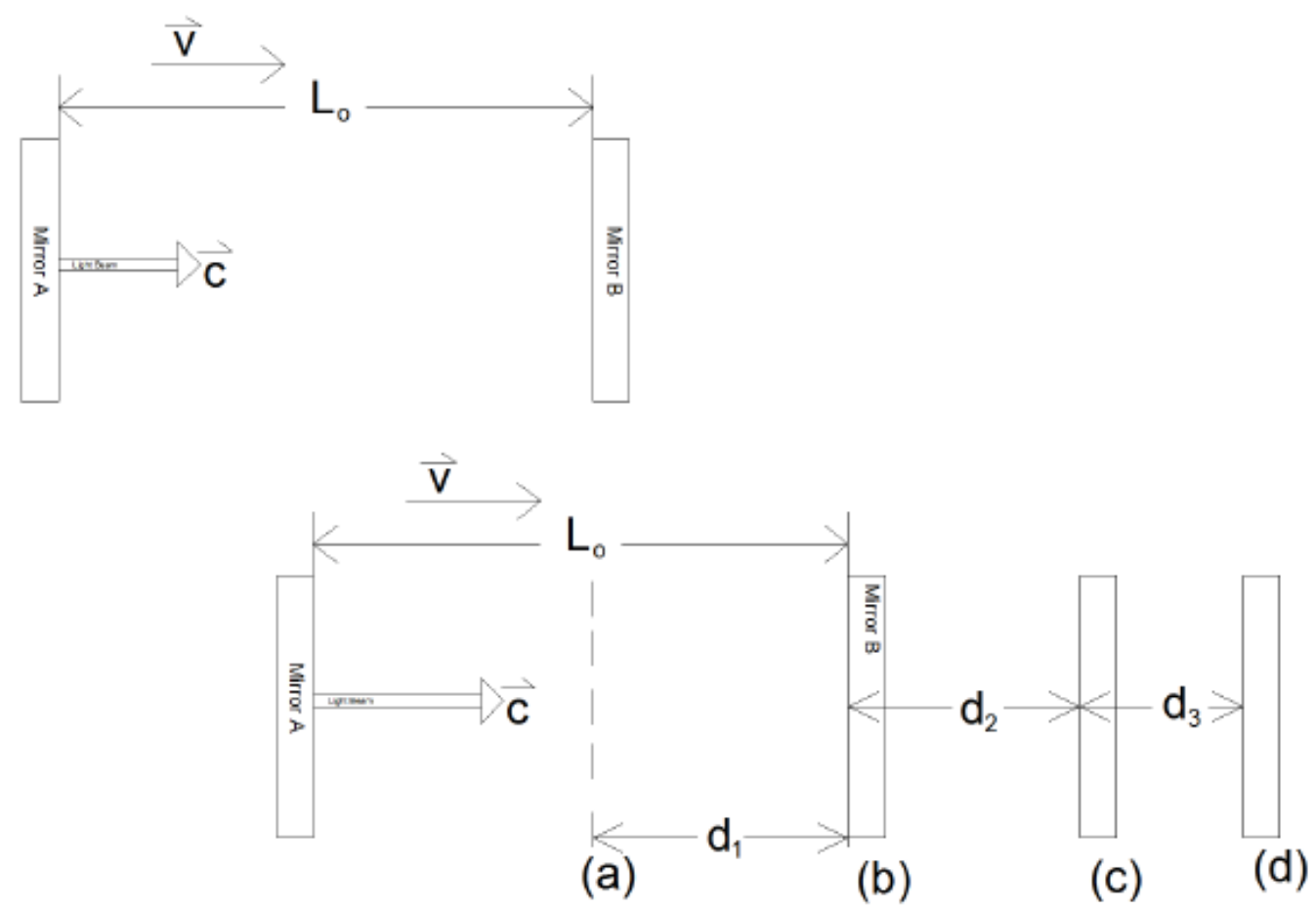

Figure 1. Diagram illustrating the iteration process used for deriving the forward period of the longitudinal light clock.

To derive the result quantitatively, we will first consider the full period of the longitudinal light clock as measured by an observer moving with the clock (frame 2). This is given simply by:

$$
T=2 L / c
$$

where $L$ is distance separating the two mirrors in the rest frame of the mirrors and $c$ is the speed of light in a vacuum.

Next we will consider the forward period of the light clock in frame 1 (where mirrors $A$ and $B$ are moving with speed $v$ ). For this derivation, we will be using the notation seen in Figure 1. Initially, the light beam will travel distance $L$ in time $t_{0}=L / c$. However, in this time mirror $B$ has receded from its initial position (a) to position (b) by the distance $d_{1}$. Given that the clock is moving with constant velocity (in frame 1 ), the distance can be given by $d_{1}=v \cdot t_{0}$. The light beam then travels for a time $t_{1}=d_{1} / c$ to reach mirror $B$ at position (b), however when the beam reaches position (b) the mirror will have receded by distance $d_{2}$ to position (c). This new distance can be calculated by $d_{2}=$ $v \cdot t_{1}$. The light beam then travels for an additional time $t_{2}=d_{2} / c$ to catch up to mirror $B$. During this time, mirror $B$ has once again receded by a distance $d_{3}=v \cdot t_{2}$ to position (d). The time that the light beam takes to reach position (d) can be given by $t_{3}=d_{3} / c$. This will then continue on, a recurring convergent process, until the light beam reaches mirror $B$. If we sum this iteration, we can add up the time terms to the total time that the light beam takes to move forward from mirror $\mathrm{A}$ to mirror $\mathrm{B}$.

$$
T_{\text {forward }}=\sum t_{n}
$$

which can be rewritten as

$$
T_{\text {forward }}=\frac{L}{c}+\frac{d_{1}}{c}+\frac{d_{2}}{c}+\frac{d_{3}}{c}+\ldots
$$

With substitutions of the distance we obtain:

$$
T_{\text {forward }}=\frac{L}{c}\left\{1+\frac{v}{c}+\left(\frac{v}{c}\right)^{2}+\left(\frac{v}{c}\right)^{3}+\cdots\right\}
$$

This can be written as the geometric series 


$$
T_{\text {forward }}=\frac{L}{c} \sum_{n=0}^{\infty}\left(\frac{v}{c}\right)^{n}
$$

Since the light clock is moving in frame 1 , a simple correction must be made for relativistic velocities-the proper length $L$ must be replaced by $L / V$ due to length contraction. Making this change and summing Equation (5) gives,

$$
T_{\text {forward }}=\frac{L}{\gamma(c-v)}
$$

To derive the time required for the light beam to travel from mirror $B$ to mirror $A$, we will use the same considerations and statements as in the previous derivation with the exception that $v$ is replaced with $-v$ (mirror $A$ is traveling in the direction against that of the light beam). The reverse time is then written as

$$
T_{\text {reverse }}=\frac{L}{c} \sum_{n=0}^{\infty}\left(\frac{-v}{c}\right)^{n}
$$

which when evaluated and adjusted for relativistic velocities yields

$$
T_{\text {reverse }}=\frac{L}{\gamma(c+v)}
$$

The next step is to verify that the addition of $T_{\text {forward }}+T_{\text {reverse }}=T_{\text {total }}$, where $T_{\text {total }}$ is the total period seen by the observer in frame 1. By doing this we end up with

$$
T_{\text {total }}=\frac{2 L \cdot c}{\gamma\left(c^{2}-v^{2}\right)}=\frac{2 L \gamma}{c}
$$

We can then substitute in the period seen by the observer in the space ship (frame 2), in which the light clock is at rest, to obtain:

$$
T_{\text {total }}=T \gamma \text { or } T^{\prime}=T_{0} \gamma
$$

As one can see, this is indeed the formula for time dilation in special relativity due to near light speeds. This result agrees with the result derived from the transverse light clock.

\section{IMPLICATIONS FOR THE FAILURE OF SIMULTANEITY}

The concept of failure of simultaneity is the result of two observers in different frames of reference experiencing the same events at different points in time. For example, the is the classical clock synchronization scenario on a moving ship [1]. A ship is moving at speed $v$ (in frame 1) with a clock at either end. The pilot walks to the center of the ship, and sends a light pulse towards the front and rear of the ship. This light pulse will cause each clock to jump to some arranged time, say 12:00. The pilot (frame 2) will see both clocks synchronized, maintaining synchronous time intervals. However, an observer at rest in frame 1 will observe that both clocks are in fact not synchronized. This is known as the failure of simultaneity.

We can use the equations for $T_{\text {forward }}$ and $T_{\text {reverse }}$ (for brevity these will be referred to as $T_{f}$ and $T_{r}$, respectively) derived in the previous section to explain the example above. Let's start by assuming that the pilot (frame 2) measures the distance between the clocks to be $2 L$. According to the observer in frame 1, when the pilot of the ship sends a light pulse in both directions, the forward and reverse light pulses will take times $T_{f}$ and $T_{r}$ respectively. Since the frame 1 observer sees $T_{f}>T_{r}$, he says the clocks are not synchronized. However in frame 2 on board ship, the pilot is at rest with respect to both clocks so he observes the light pulses to reach both clocks simultaneously.

\section{CONCLUSION AND ZENO'S PARADOX}

We were able to derive the formula for time dilation used in special relativity for the longitudinal light clock, and the result agreed with the result from the transverse light clock. In addition, the derivation and individual time results $\left(T_{f}\right.$ and $\left.T_{r}\right)$ provided insight into the solution of some paradoxes that fall under the failure of simultaneity.

One of the paradoxes proposed by Zeno of Ela is that of Achilles and the Tortoise. The paradox states:

....in a race the quickest runner can never overtake the slowest, since the pursuer must first reach the point whence the pursued started, so that the slower must always hold a lead. Aristotle, Physics, VI: 9, $239 b 15$ [4] 
In this paradox, Achilles is chasing a totoise, and the tortoise is given a head start. To catch up to the tortoise, Achilles must first run to the location where the tortoise was when Achilles began running. By the time that Achilles reaches that position, the tortoise will have already moved forward some distance. Achilles must then run to the new location of the tortoise, and so on. This distance eventually becomes infinitesimally small, but Achilles never catches up to the tortoise due to the fact that he must first run to the location where the tortoise was. This is obviously a paradox since we know that Achilles is faster than the tortoise-he will eventually catch up and overtake the tortoise [5]. In both the case of Zeno's paradox and the longitudinal light clock, the solution (whether to the paradox or the period) was obtained by summing an infinite series.

\section{ACKNOWLEDGEMENTS}

We would like to thank Professor Patrick Moylan for his help and support on this project. In addition we would like to thank him for suggesting the connection to Zeno's paradox as well as for the title of the paper.

\section{REFERENCES}

1. Feynman, Leighton, and Sands. The Feyman Lectures on Physics (Pearson Addison Wesley, 2006).

2. Halliday, Resnick, and Walker. Fundamentals of Physics, $8^{\text {th }}$ edition (Wiley, 2008).

3. Francis, Gregory, and Kirkpatrick, Larry. Physics: A World View, $6^{\text {th }}$ edition (Brooks/Cole, 2007).

4. Aristotle. Physics. Translated by R.P. Hardie and R.K. Gaye

5. "Zenos Paradoxes," in: Stanford Encyclopedia of Philosophy (2002).

\section{www.uni.edu/ajur}

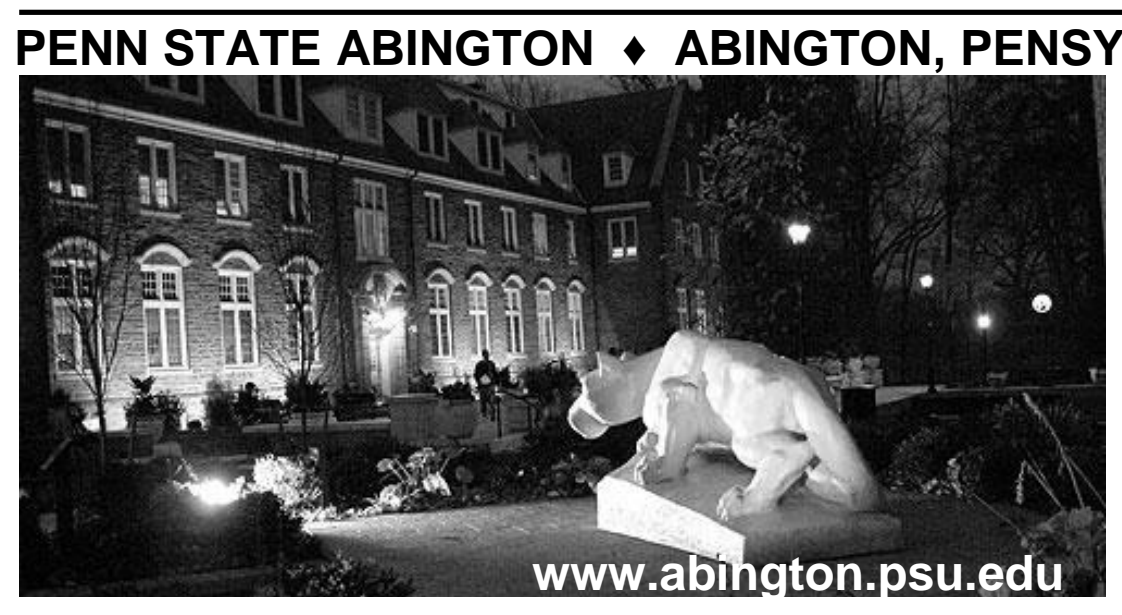

The college offers a full range of academic and student support services, including a lower-division college, an honors program, an upper-division Schreyer Honors College program, internships, undergraduate research with faculty, state-of-the-art computer facilities, a learning center, a wide range of student clubs and organizations, varsity and club sports, and a career development center. For additional information, please contact the Office of Admissions, Penn State Abington, 1600 Woodland Road, Abington, PA 19001; 215-881-7600.
Penn State's

Abington College, located on forty-five picturesque acres in suburban Philadelphia, offers a small-university environment within the context of a major university. Students beginning their academic careers at Penn State Abington have the flexibility to complete their degrees at Abington, University Park, or at one of the several other Penn State locations around the state. 